\title{
Direct Projections from Cochlear Nuclear Complex to Auditory Thalamus in the Rat
}

\author{
Manuel S. Malmierca, ${ }^{1}$ Miguel A. Merchán, ${ }^{1}$ Craig K. Henkel, ${ }^{2}$ and Douglas L. Oliver ${ }^{3}$ \\ ${ }^{1}$ Laboratory for the Neurobiology of Hearing, Institute for Neuroscience of Castilla y León and Faculty of Medicine, \\ University of Salamanca, 37007 Salamanca, Spain, ${ }^{2}$ Wake Forest University School of Medicine, Department of \\ Neurobiology and Anatomy, Winston-Salem, North Carolina 27157-1010, and 3University of Connecticut Health Center, \\ Department of Neuroscience, Farmington, Connecticut 06030-3401
}

It is known that the dorsal cochlear nucleus and medial geniculate body in the auditory system receive significant inputs from somatosensory and visual-motor sources, but the purpose of such inputs is not totally understood. Moreover, a direct connection of these structures has not been demonstrated, because it is generally accepted that the inferior colliculus is an obligatory relay for all ascending input. In the present study, we have used auditory neurophysiology, double labeling with anterograde tracers, and retrograde tracers to investigate the ascending projections of the cochlear nuclear complex. We demonstrate that the dorsal cochlear nucleus and the small cell cap of the ventral cochlear nucleus have a direct projection to the medial division of the medial geniculate body. These direct projections from the cochlear nucleus complex bypass the

The multimodal aspects of information processing are a newly emerging aspect of auditory function in the dorsal cochlear nucleus (DCN). Although the DCN is part of the first synaptic station in the auditory pathway, the cochlear nucleus complex (CNC), and receives heavy synaptic inputs from the cochlea, it also receives direct inputs from the somatosensory system (Itoh et al., 1987; Weinberg and Rustioni, 1987; Wright and Ryugo, 1996), vestibular system (Burian and Gstoettner, 1988), and pontine inputs (Ohlrogge et al., 2001). These multimodal inputs may be important for the modulation of DCN activity during movement of the animal, especially during movement of the pinnas (Young et al., 1995; Kanold and Young, 2001).

Integration of auditory, somatosensory, and visual-motor inputs has also been implicated for other parts of the auditory system. For example, the medial geniculate body (MGB) has long been known to receive somatosensory and visual-motor inputs (Morest, 1965; Jones and Burton, 1974; Oliver and Hall, 1978; Berkley et al., 1980). Visual-motor, gaze-control structures, such as the superior colliculus (du Lac and Knudsen, 1990; Guitton,

\footnotetext{
Received July 22, 2002; revised Oct. 2, 2002; accepted Oct. 3, 2002.

This work was supported by the Fulbright Commission (M.A.M., M.S.M., D.L.O.), Spanish Junta de Castilla y León-Uniòn Europea (SA084/01) and Dirección General de Enseñanza Superior (BFI-2000-1296) (M.S.M., M.A.M.), and National Institutes of Health Grants DC00189 (D.L.O.) and DC00813 (C.K.H.). We thank Nell Cant, Shig Kuwada, Adrian Rees, and Brett Schofield for their suggestions and critical reading of a previous version of this manuscript. We thank Drs. D. Godfrey and D. Ryugo for suggesting references and Ignacio Plaza for his excellent technical assistance.

Correspondence should be addressed to Manuel S. Malmierca, Laboratory for the Neurobiology of Hearing, Institute for Neuroscience of Castilla y León, University of Salamanca, 37007 Salamanca, Spain. E-mail: msm@usal.es.

Copyright (C) 2002 Society for Neuroscience 0270-6474/02/2210891-07\$15.00/0
}

inferior colliculus and are widely distributed within the medial division of the medial geniculate, suggesting that the projection is not topographic. As a nonlemniscal auditory pathway that parallels the conventional auditory lemniscal pathway, its functions may be distinct from the perception of sound. Because this pathway links the parts of the auditory system with prominent nonauditory, multimodal inputs, it may form a neural network through which nonauditory sensory and visual-motor systems may modulate auditory information processing.

Key words: medial geniculate body; auditory pathways; lateral lemniscus; brachium of the inferior colliculus; nonlemniscal pathway; double labeling; dextran; multimodal information processing

1992; Freedman and Sparks, 1997), also receive auditory input via the nucleus of the brachium of the inferior colliculus (IC) (King et al., 1998; Doubell et al., 2000). Somatosensory inputs are also a prominent feature of the lateral, external cortex of the IC (Morest and Oliver, 1984).

Here we specifically focus on the axonal projections of the multimodal areas of the $\mathrm{CNC}$ in experiments that use combined neuroanatomical and neurophysiological methods in the rat. DCN and other CNC axons project directly to the medial division of the MGB (MGM) in a manner that differs remarkably from their previously known projections to the IC. Because the DCN, lateral cortex of the IC, and MGM are all interconnected and involved in multimodal integration, they represent a nonlemniscal auditory pathway whose function is likely distinct from the lemniscal auditory pathways.

A preliminary report has been published previously in abstract form (Malmierca et al., 2002).

\section{MATERIALS AND METHODS}

In this study, 15 rats with injections in the CNC (Table 1) were examined for anterograde labeling of axons. Also, a collection of four rats with horseradish peroxidase (HRP) injections and an additional rat with a biotinylated-dextran amine (BDA) injection in the MGB were examined for retrograde labeling of cells in the CNC. All experiments were performed with methods in keeping with the standards and approval of the University of Salamanca Animal Care Committee and National Institutes of Health animal care guidelines. Two groups of experiments have been performed:

Group 1. We made injections of BDA, fluorescein-dextran (FD), or tetramethylrhodamine-dextran (TRD) into physiologically defined regions of the DCN and ventral cochlear nucleus in the same animals (Table 1) as published previously (Oliver et al., 1997). An areflexive, anesthetic state was induced by intramuscular administration of ket- 
Table 1. Summary of animals and injection sites in the $\mathrm{CNC}$ subdivisions

\begin{tabular}{|c|c|c|c|c|c|}
\hline \multirow{2}{*}{$\begin{array}{l}\text { Animals } \\
(n=15)\end{array}$} & \multicolumn{5}{|l|}{${\underline{\text { Injection site }}(\mathrm{BF})^{a}}^{a}$} \\
\hline & $\mathrm{DCN}(\mathrm{kHz})$ & $\mathrm{DCN}(\mathrm{kHz})$ & AVCN (kHz) & Cap area $(\mathrm{kHz})$ & PVCN (kHz) \\
\hline R-005 & TRD (15) & & & & $\mathrm{FD}+\mathrm{BDA}(15)$ \\
\hline $\mathrm{R}-021$ & TRD (11) & & $\mathrm{FD}+\mathrm{BDA}(11)$ & & \\
\hline R-034 & TRD (6) & & & $\mathrm{FD}+\mathrm{BDA}$ medial part $^{b}$ & $\mathrm{FD}+\mathrm{BDA}(1.5)$ \\
\hline R-067 & TRD (13) & & & & $\mathrm{FD}+\mathrm{BDA}(13.5)$ \\
\hline R-109 & TRD (2.5) & & & & \\
\hline R-111 & TRD (1.9) & & & & \\
\hline $\mathrm{R}-112$ & TRD (3.5) & $\mathrm{FD}+\mathrm{BDA}(6.5)$ & & & \\
\hline $\mathrm{R}-114$ & $\mathrm{FD}+\mathrm{BDA}(2.5)$ & & & TRD lateral part ${ }^{b}$ & TRD (4.8) \\
\hline R-117 & $\mathrm{FD}+\mathrm{BDA}(4.5)$ & & & & \\
\hline R-119 & $\mathrm{FD}+\mathrm{BDA}(27)$ & TRD (13.5) & & & \\
\hline $\mathrm{R}-120$ & $\mathrm{FD}+\mathrm{BDA}(5)$ & & TRD (5.2) & & \\
\hline $\mathrm{R}-121$ & $\mathrm{FD}+\mathrm{BDA}(1.7)$ & TRD (1.8) & & & \\
\hline $\mathrm{R}-122$ & TRD (31) & & $\mathrm{FD}+\mathrm{BDA}(30)$ & & \\
\hline R-135 & TRD (3.5) & & & & \\
\hline $\mathrm{R}-231$ & TRD (29) & $\mathrm{FD}+\mathrm{BDA}(6.3)$ & & & \\
\hline
\end{tabular}

${ }^{a} \mathrm{BF}$, Best frequency.

${ }^{b}$ Injections in the marginal shell (cap area) that encroach on the PVCN.

amine $(57 \mathrm{mg} / \mathrm{kg})$ and xylazine $(8.6 \mu \mathrm{g} / \mathrm{kg})$ and maintained with the same compounds. Animals were held in a stereotaxic frame and monitored by observing respiration rate and reflexes. A craniotomy was performed over the cerebellum, and a part of the lateral and floccular cerebellum was aspirated to expose the CNC.

Extracellular recordings in response to acoustic stimulation allowed the determination of best frequency (sound frequency to which the neurons respond with the lowest intensity) at the injection sites in the right DCN and right anteroventral cochlear nucleus (AVCN) and posteroventral cochlear nucleus (PVCN). Animals were placed in a doublewall sound attenuation chamber. Earphones in a sealed enclosure were coupled to the ear bars of the stereotaxic frame, and pure tones were delivered by digital stimulus generators under the control of a computer system (Rees et al., 1997). Recordings were made with glass micropipettes $(10-40 \mu \mathrm{m}$ tips) filled with injection solutions for anterograde transport. One injection electrode contained $10 \%$ TRD dissolved in saline; the other electrode contained a mixture of $10 \%$ BDA and $10 \%$ FD in saline. Once the desired site was found, the dextrans were injected by iontophoresis (2-6 $\mu \mathrm{A}$ for $5-24 \mathrm{~min})$.

Seven to $10 \mathrm{~d}$ after the injections, the brains were perfusion fixed and prepared for light microscopy. Although under deep surgical anesthesia, the animals were perfused transcardially with a buffered washout solution $(2 \%$ sucrose in $0.12 \mathrm{M}$ phosphate buffer, $\mathrm{pH} 7.4$, containing $0.05 \%$ lidocaine with $0.004 \% \mathrm{CaCl}_{2}$ ) followed by a $4 \%$ paraformaldehyde fixative solution. After fixation, decapitation, and dissection, the brain tissue was cryoprotected in $30 \%$ sucrose and sectioned in the transverse plane into 35 - or $50-\mu \mathrm{m}$-thick slices on a freezing microtome. Adjacent sections underwent avidin-biotin complex histochemistry for BDA (black reaction) followed by immunohistochemistry with antisera to rhodamine, biotinylated secondary antisera, and avidin-biotin histochemistry (red reaction). Every third or fourth section was used for Nissl counterstain.

Group 2. Iontophoretic injections of HRP were made into the rat $\operatorname{MGM}(n=2)$ and ventral MGB (MGV; $n=2)$ and revealed as described previously (Merchán et al., 1994). In an additional case, we made an injection of FD and another injection of TRD in the MGV in the same rat and revealed as described above.

Camera lucida drawings were made with the aid of a drawing tube attached to a Leica (Wetzlar, Germany) DMRB microscope. Photomicrographs were made with an Olympus Optical (Tokyo, Japan) DP10 digital camera.

\section{RESULTS}

Our results are based on two different groups of complementary experiments. One group had one or two injections of anterograde tracers in the DCN, AVCN, or PVCN (Table 1). A second group had injections of the retrograde tracer in the MGB.

\section{Anterograde labeling of the CNC-MGB projection}

After injections in the DCN (Fig. 1a) (Table 1), labeled axons formed a distinct lamina in the contralateral IC (Fig. 1b). The same injection produced axonal labeling throughout the contralateral MGM in all cases (Figs. 1-4). The labeling formed a continuous rostrocaudal plexus of terminals (Fig. $2 c-f$ ). Terminal boutons and axonal swellings in passing were found throughout the MGM (Figs. 1c, 2f, 4a). No labeling was found in the MGV in any of the experiments (Figs. 2-4). In two cases, some terminal fields were observed at the medial edge of the contralateral dorsal $\mathrm{MGB}$, an area that may correspond to the suprageniculate nucleus of the MGB.

Fibers to the MGM followed the dorsal acoustic stria in the tegmentum ventral to the IC, contralateral to the DCN injection. They continued to the MGM with the brachium of the IC, giving collaterals and terminal boutons to its nucleus, where they formed a dorsoventrally oriented band of terminals. In a few cases, there were some labeled axons that terminated in the ipsilateral MGM, where they were discontinuous and patchy along the rostrocaudal axis. No retrogradely labeled neurons were found in the IC contralateral to the injection into the $\mathrm{CNC}$ that could account for the labeling seen in the contralateral MGM. When a second tracer was placed in the lateral or medial edges of the PVCN, including the marginal shell or cap area of the same animal on the same side (Table 1) (Fig. $2 b)(n=2)$, additional labeling was seen in the contralateral MGM (Fig. $2 f$, asterisk). If a second tracer was placed in the AVCN of the same animal on the same side (Fig. $4 a)(n=3)$, no labeled axons were found in the MGB (Fig. 4b,c). In the cases with tracer injections involving very caudal AVCN and PVCN (Table 1), there may have been some spread into the DCN, so that a projection from these regions could not be confirmed with anterograde tracers.

A fundamental property of the auditory nervous system is its tonotopic organization. Therefore, a key issue is whether the 

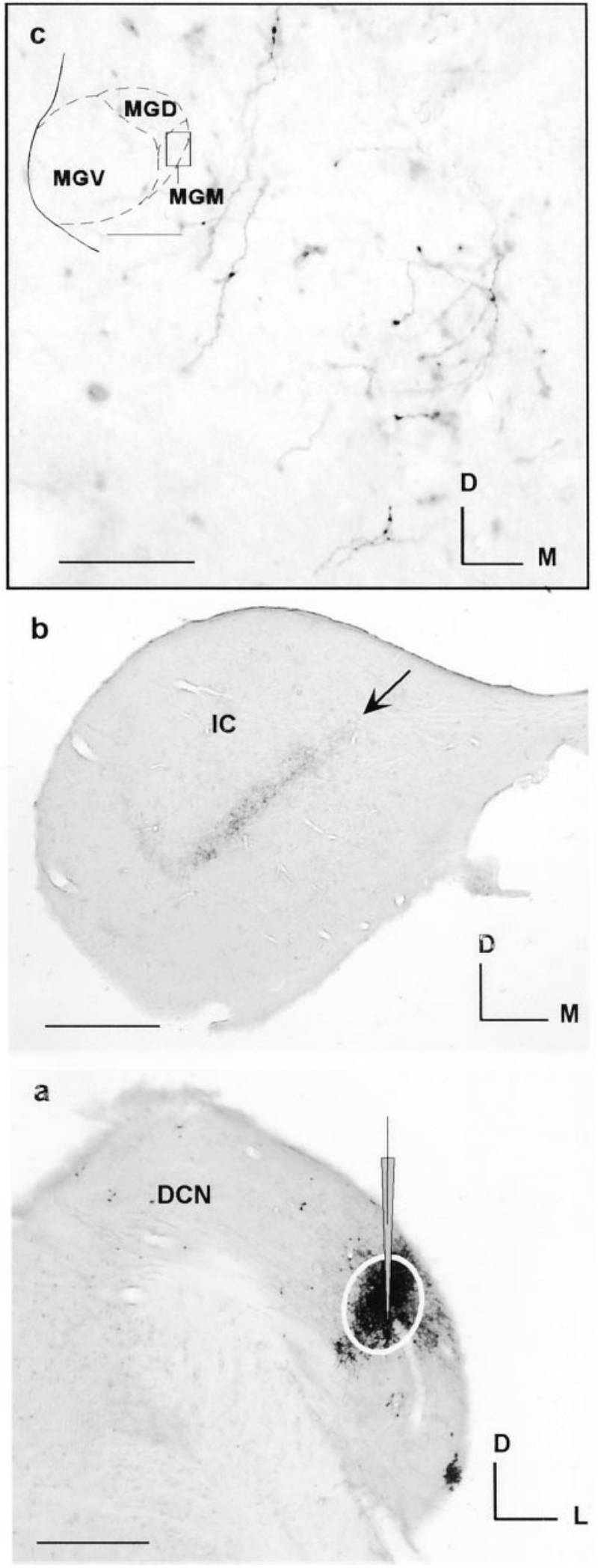

Figure 1. Axons in the medial division of the MGM labeled by anterograde transport of dextran after an injection in the DCN (Table 1, R-117). $a$, Micrograph of the injection (micropipette, white circle) at best frequency $(4.5 \mathrm{kHz}) . b$, Low-magnification micrograph of the IC showing laminar axons in the central nucleus (arrow). c, High-magnification micrograph of labeled axons in MGM (see inset, drawing of MGB, dashed circle). Scale bars: $a, b, 500 \mu \mathrm{m} ; c, 50 \mu \mathrm{m}$. $D$, Dorsal; $M$, medial; $L$, lateral. projection from the $\mathrm{CNC}$ to the MGM is tonotopic. Regardless of the best frequency of the injections in the CNC, the terminal fields overlapped in the MGM. In those cases in which the two injections had different best frequencies $(n=5)$ (Figs. 2, 3), the terminal fields observed in the MGM overlapped (Figs. $2 f, 3 c$ ), although two distinct frequency-band laminas were present as expected in the IC (Figs. $2 g$, 3b, arrows). Two injections with the same best frequency also resulted in overlapping projections to the MGM, and in these cases, only one frequency-band lamina was present in the IC ( $n=2$; data not shown). Therefore, we conclude that the CNC-MGM projection is nontopographic, which is consistent with the broad frequency tuning in MGM neurons (Bordi and LeDoux, 1994).

\section{Retrograde labeling of the CNC-MGM projection}

To identify the cell types involved and confirm that the CNCMGM projection was not caused by retrograde-anterograde labeling from the auditory neocortex (Winer and Larue, 1987; Rouiller and Welker, 1991; Feliciano et al., 1995; Weedman and Ryugo, 1996), a second group of experiments was performed with injections of HRP in the MGM $(n=2)$ (Fig. 5) and MGV $(n=$ 2). The distribution of retrogradely labeled cells in the CNC after injections in the MGM (Fig. 5a) showed labeled neurons in the contralateral DCN in the deep layer (Fig. $5 b, c$ ) or in the pyramidal cell area. Some of these labeled cells were likely pyramidal cells based on their location, shape, and the perikaryal size (Osen, 1969; Blackstad et al., 1984). Other, more abundant, labeled DCN cells were of the giant type (Fig. 5c). At the borders of the PVCN, some labeled neurons could be small cells in the marginal shell or cap area. On the ipsilateral side, only a small number of cells (two to three) were found in one case on the PVCN border. In one case, neurons were also observed in the nucleus $\mathrm{X}$, which is related to the vestibular complex (Fig. $5 b$ ). We did not record the best frequency at the injection site in these experiments, but a comparison of the labeled cells with published tonotopic maps in the rat (Ryan et al., 1988; Rouiller et al., 1992) suggests that the projection to the MGM arises from the middle- and highfrequency regions of the DCN.

In the two cases with HRP injections into the MGV, no labeled cells were found in any division of the CNC. Likewise, two injections of the MGV in the same animal (best frequencies of 6 and $12-15 \mathrm{kHz}$, respectively) with FD and TRD produced no labeled cells in any CNC region. However, labeled neurons were found in the IC in two separate laminas, and a few scattered cells were apparent in the nuclei of the lateral lemniscus and superior olivary complex (Aitkin and Phillips, 1984).

\section{DISCUSSION}

Our results unambiguously demonstrate the existence of a CNCMGM projection. The CNC-MGM projection may parallel the conventional, lemniscal auditory pathway. This nonlemniscal auditory pathway projects directly to the thalamus, may involve multiple levels of the auditory brainstem, including the cortex of the IC, and may facilitate multimodal modulation of the auditory neocortex. It differs from the central acoustic tract of Ramón y Cajal (1902), because he believed that the CNC projected to all divisions of the MGB. It also differs from the central acoustic tract of the bat, whose origin is near the superior olive and whose projections are strictly ipsilateral (Casseday et al., 1989). The CNC-MGM pathway in the present study is a crossed pathway. It is currently unknown whether a homologous pathway to the central acoustic tract of the bat exists in the rat. 
Figure 2. Distribution of axonal labeling after two separate injections in the DCN and PVCN (Table 1, R-034). $a$, Camera lucida drawing of a TRD injection (red, red pipette) in $6 \mathrm{kHz}$ DCN. $b$, Injection of fluorescein-dextran and biotinylated dextran (FD + BDA; black, yellow pipette) into the PVCN in the same animal at $1.5 \mathrm{kHz}$ best frequency. $c$, Plots of serial sections showing the location of TRD axons (red) that form a continuous plexus of terminal boutons confined to the MGM and overlap with BDA axons (black). $d$, Camera lucida drawing of a Nissl-stained section through the MGB, also shown as a photomicrograph in $d^{\prime} . e$, Adjacent section to that shown in $d^{\prime}$. e, Photomicrograph of the axons in MGM also shown at higher magnification (red window) in $f$. Observe the overlap of red and black axons (asterisk) as opposed to the two distinct laminas seen in the IC $(g, a r$ rows). Scale bars: $a-e, g, 500 \mu \mathrm{m} ; f, 100$ $\mu \mathrm{m}$. $D$, Dorsal; $M$, medial; $L$, lateral.

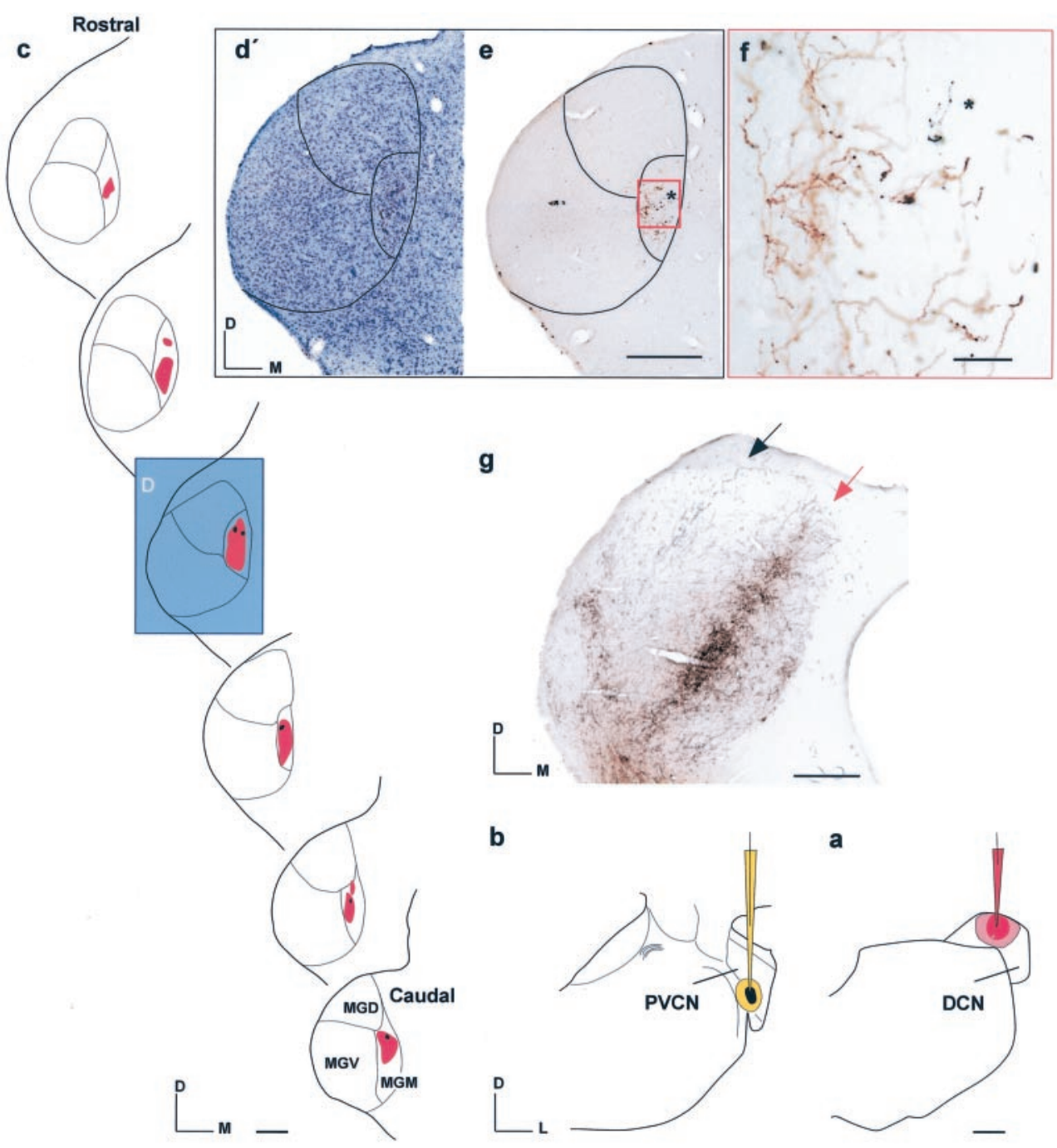

The direct CNC-MGM pathway may be found in diverse species. Interestingly, 25 years ago, Strominger et al. (1977) reported the direct $\mathrm{CNC}-\mathrm{MGM}$ projection in primates after an experiment on a single chimpanzee. There, degenerating axons in the MGB were identified after a lesion in the cochlear nucleus and adjacent medulla. That lesion could have injured axons from structures other than the cochlear nucleus. But the likelihood that this early observation was accurate is further increased by recent observations that the direct CNC-MGM pathway is present in the ferret (B. Schofield and N. Cant, unpublished observations), guinea pig (B. Schofield, personal communication), and gerbil (N. Cant, personal communication), and the by present results. Thus, rodent, carnivore, and primate species may share the same nonlemniscal pathway shown here in rat. The cell types used in the direct CNC-MGM pathway may determine whether the pathway has a common function across species and may provide clues to its function.

Pyramidal and giant cells participate in the direct CNC-MGM pathway, and both have a type IV frequency-response map that allows the detection of spectral notches introduced by the pinnas (Yu and Young, 2000). These notches provide monaural spectral cues that may be used for the localization of sounds in the vertical plane. Because the neurons in the DCN receive somatosensory input from the dorsal column nuclei and the trigeminal sensory nucleus (Itoh et al., 1987; Young et al., 1995; Shore et al., 2000), proprioceptive information from the pinnas appears to modulate the activity of these cells during movements of the pinnas (Kanold and Young, 2001). The cells of the small cell cap of the CNC also participate in the CNC-MGM projection. These neurons possess a very wide dynamic range, $\leq 98 \mathrm{~dB}$ (Ghoshal and Kim, 1996), suggesting that the direct CNC-MGM pathway would have an equally broad dynamic range.

The conventional lemniscal pathway (i.e., the auditory pathway used for the perception of sound) may parallel the direct CNCMGM pathway. The lemniscal pathway originates with bushy and stellate neurons of the AVCN, whose axons project to the superior olivary complex and IC, respectively, and continues to the MGV. Binaural interactions must be a property of the lemniscal pathway, because they first take place in the superior olivary complex. Moreover, frequency discrimination and spectral processing are most likely functions of the lemniscal pathway only, because the nonlemniscal pathway is demonstrated here to lack topographic organization. Other DCN projections to the IC also must be included in the lemniscal pathway, because these projections are highly topographic and easily related to the tonotopic organization of the nucleus (present results) (Oliver, 1985).

The present results suggest that a nonlemniscal pathway is composed of the DCN, MGM, and lateral cortex and nucleus of 

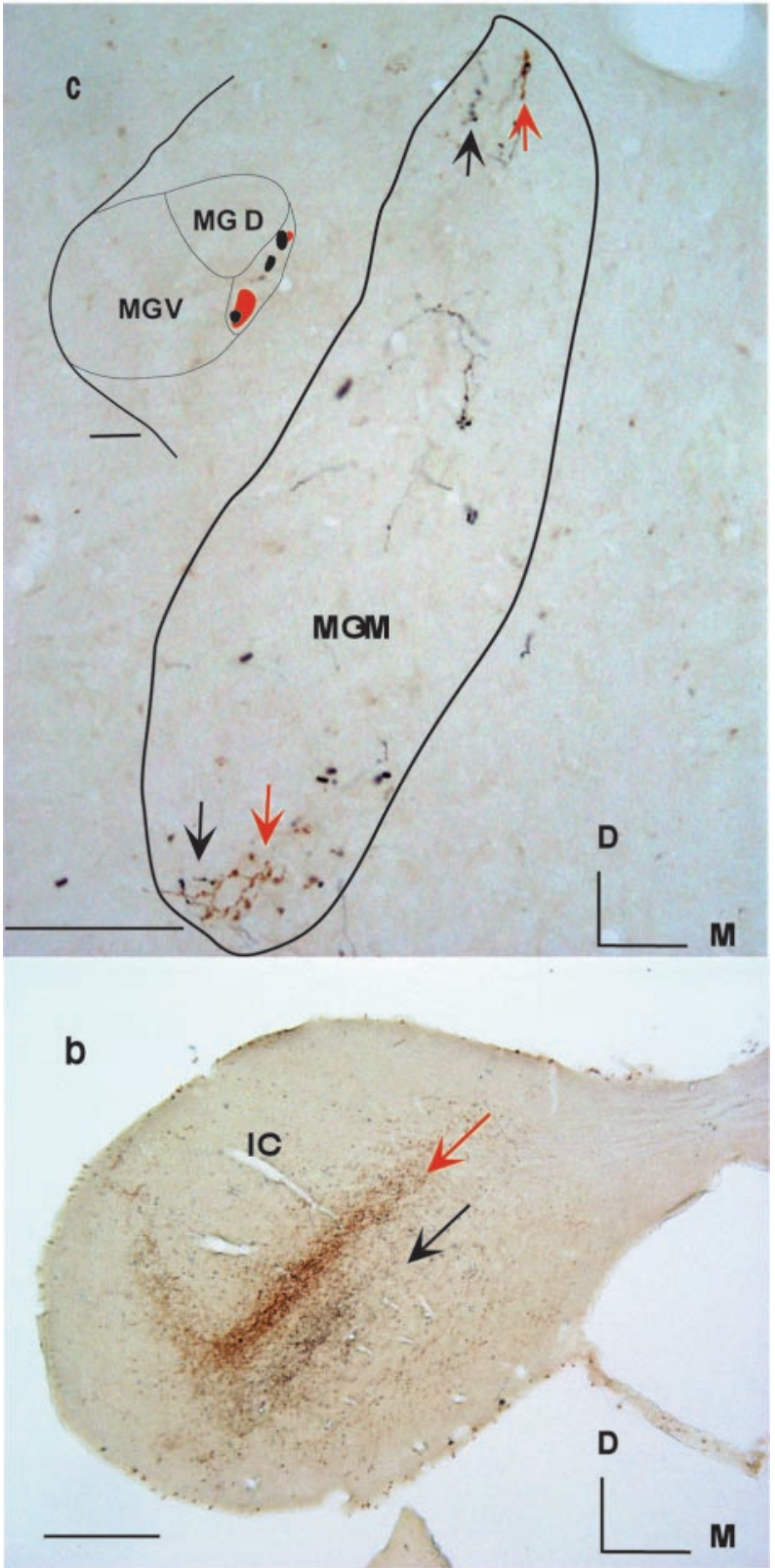

a

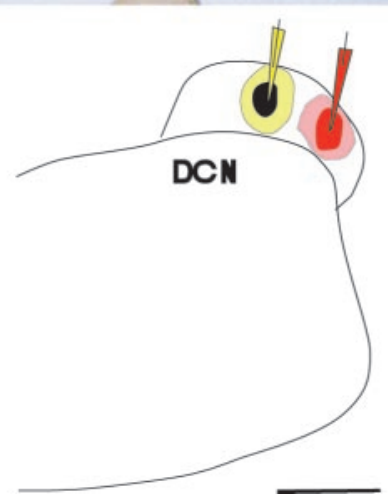

Figure 3. Labeling in the MGM (c) and IC (b) after two injections in the $\operatorname{DCN}(a)$ one octave apart, $27 \mathrm{kHz}$ (black-yellow, pipette) and $13.5 \mathrm{kHz}$ (red, pipette) (Table 1, R-119). Nonoverlapping laminas are labeled in the IC ( $b$, red and black arrows), whereas contiguous and overlapping axonal termination is seen in the MGM ( $c$, arrows within outline of MGM). Scale bars: $a, b, 500 \mu \mathrm{m} ; c, 100 \mu \mathrm{m}$; inset in $a, 250 \mu \mathrm{m}$. $D$, Dorsal; $M$, medial; $L$, lateral.
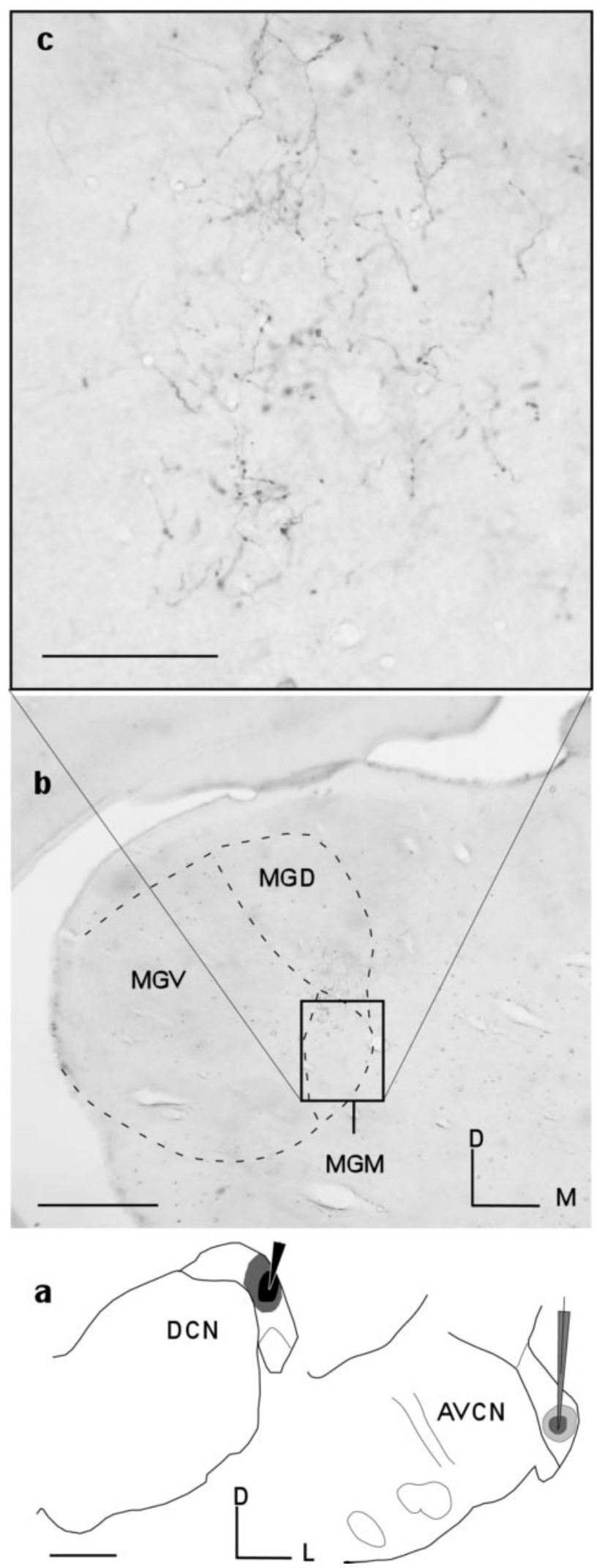

Figure 4. Axonal labeling in the $\operatorname{MGM}(b, c)$ after two injections $(a)$ at the same best frequency, $11 \mathrm{kHz}$, one in the DCN (black, pipette) and the other in the AVCN (gray, pipette) (Table 1, R-021). b, Labeled axons in the medial geniculate body are seen only from the injection in the DCN in the lower-power micrograph ( $b, \mathrm{MGB}$, dashed lines) and the enlargement $(c)$. Scale bars: $a, b, 500 \mu \mathrm{m} ; c, 100 \mu \mathrm{m}$. $D$, Dorsal; $M$, medial; $L$, lateral. 
a

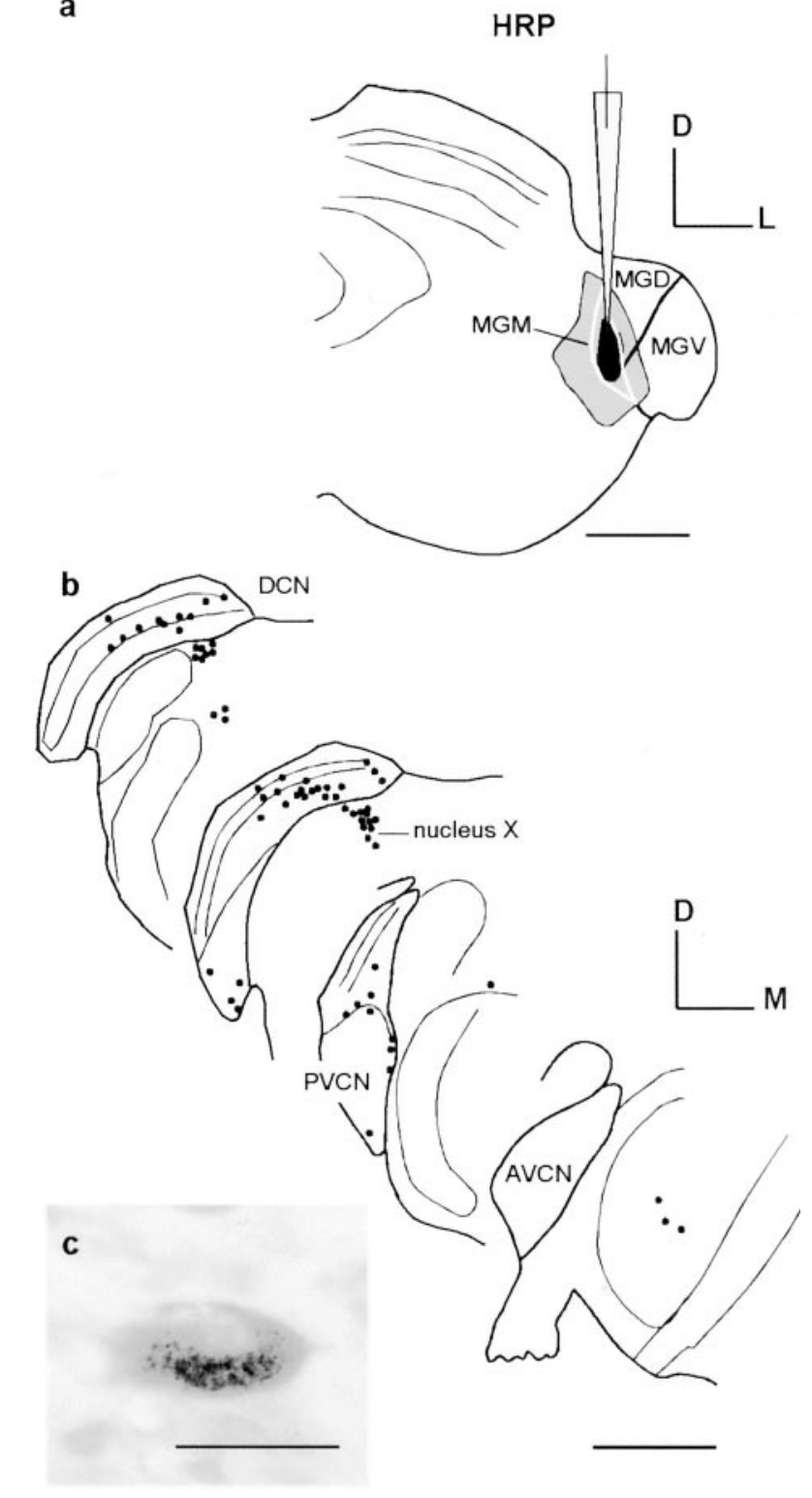

Figure 5. Retrograde labeling of the projection from the cochlear nuclear complex to the medial geniculate body. $a$, Injection of HRP in the MGM (black, pipette). $b$, Labeling in the DCN and PVCN (black dots). $c$, A typical example of labeled giant cell. Scale bars: $a, b, 1 \mathrm{~mm} ; c, 50 \mu \mathrm{m}$. $D$, Dorsal; $M$, medial; $L$, lateral.

the brachium of the IC. The MGM has long been suspected to have a unique role in auditory processing because of multisensory and visual-motor inputs, including spinal cord inputs and the superior colliculus (Wepsic, 1966; Guitton, 1992; Freedman and Sparks, 1997; Shiroyama et al., 1999). Like the DCN and the MGM, the lateral cortex of the IC also receives somatosensory inputs from the spinal cord and dorsal column nuclei (Morest and Oliver, 1984). It is tempting to speculate that pinnas and head and eye movements all influence the activity of the nonlemniscal pathway. The nonlemniscal pathway may provide a broad spectral tuning to neurons in the MGM consistent with the convergence of the CNC-MGM pathway shown here. In addition, the nonlemniscal pathway is likely to function over a wide range of intensities, consistent with the present findings of direct small cell cap projections to the MGM.
Information in the lemniscal and nonlemniscal pathways may travel over nearly the same time course. First-spike latencies reported for the IC average 9-11 msec (ranging from 6.2 to 77 msec) (Rees and Moller, 1983; Palombi and Caspary, 1996), whereas those for the MGM average $10 \mathrm{msec}$ but can be as short as $7 \mathrm{msec}$ (Bordi and LeDoux, 1994). A similar range of latencies suggests that lemniscal and nonlemniscal pathways could influence each other and do so more readily than assumed previously.

The lemniscal and nonlemniscal pathways may converge in the auditory neocortex. MGM axons terminate primarily in layers 1 and 6, whereas the MGV terminates in layer 4 (Ryugo and Killackey, 1974; Oliver and Hall, 1978; Herkenham, 1980; Cetas et al., 1999; Linke and Schwegler, 2000; Smith and Spirou, 2002). Little is known about the functional organization of layer 1, but Cauller and Connors (1992) demonstrated that afferent synapses on most distal dendrites of the pyramidal cortical neurons exert a powerful influence on their firing probability. Background synaptic input may modulate the gain of synaptic inputs to pyramidal cells (Chance et al., 2002). Thus, activity in the nonlemniscal pathway related to pinnas and head and eye movements may modulate the gain of sound-evoked activity from the lemniscal pathway through distal synaptic input on pyramidal cortical neurons.

\section{REFERENCES}

Aitkin LM, Phillips SC (1984) Is the inferior colliculus an obligatory relay in the cat auditory system? Neurosci Lett 44:259-264.

Berkley KJ, Blomqvist A, Pelt A, Flink R (1980) Differences in the collateralization of neuronal projections from the dorsal column nuclei and lateral cervical nucleus to the thalamus and tectum in the cat: an anatomical study using two different double-labeling techniques. Brain Res 202:273-290.

Blackstad TW, Osen KK, Mugnaini E (1984) Pyramidal neurones of the dorsal cochlear nucleus: a Golgi and computer reconstruction study in cat. Neuroscience 13:827-854.

Bordi F, LeDoux JE (1994) Response properties of single units in areas of rat auditory thalamus that project to the amygdala. Exp Brain Res 98:261-274.

Burian M, Gstoettner W (1988) Projection of primary vestibular afferent fibres to the cochlear nucleus in the guinea pig. Neurosci Lett 84:13-17.

Casseday JH, Kobler JB, Isbey SF, Covey E (1989) Central acoustic tract in an echolocating bat: an extralemniscal auditory pathway to the thalamus. J Comp Neurol 287:247-259.

Cauller JL, Connors BW (1992) Functions of very distal dendrites: experimental and computational studies of layer I synapses on neocortical pyramidal cells. In: Single neuron computation (McKenna T, Davis J, Zornetzer SF, eds), pp 199-229. San Diego: Academic.

Cetas JS, De Venecia RK, McMullen NT (1999) Thalamocortical afferents of Lorente de No: medial geniculate axons that project to primary auditory cortex have collateral branches to layer I. Brain Res 830:203-208.

Chance F, Abbott L, Reyes A (2002) Gain modulation from background synaptic input. Neuron 35:773-783.

Doubell TP, Baron J, Skaliora I, King AJ (2000) Topographical projection from the superior colliculus to the nucleus of the brachium of the inferior colliculus in the ferret: convergence of visual and auditory information. Eur J Neurosci 12:4290-4308.

du Lac S, Knudsen EI (1990) Neural maps of head movement vector and speed in the optic tectum of the barn owl. J Neurophysiol 63:131-146.

Feliciano M, Saldaña E, Mugnaini E (1995) Direct projections from the rat primary neocortex to nucleus sagulum, paralemniscal regions, superior olivary complex and cochlear nuclei. Aud Neurosci 1:287-308.

Freedman EG, Sparks DL (1997) Activity of cells in the deeper layers of the superior colliculus of the rhesus monkey: evidence for a gaze displacement command. J Neurophysiol 78:1669-1690.

Ghoshal S, Kim DO (1996) Marginal shell of the anteroventral cochlear nucleus: intensity coding in single units of the unanesthetized, decerebrate cat. Neurosci Lett 205:71-74.

Guitton D (1992) Control of eye-head coordination during orienting gaze shifts. Trends Neurosci 15:174-179.

Herkenham M (1980) Laminar organization of thalamic projections to the rat neocortex. Science 207:532-535.

Itoh K, Kamiya H, Mitani A, Yasui Y, Takada M, Mizuno N (1987) Direct projections from the dorsal column nuclei and the spinal trigeminal nuclei to the cochlear nuclei in the cat. Brain Res 400:145-150. 
Jones EG, Burton H (1974) Cytoarchitecture and somatic sensory connectivity of thalamic nuclei other than the ventrobasal complex in the cat. J Comp Neurol 154:395-432.

Kanold PO, Young ED (2001) Proprioceptive information from the pinna provides somatosensory input to cat dorsal cochlear nucleus. J Neurosci 21:7848-7858.

King AJ, Jiang ZD, Moore DR (1998) Auditory brainstem projections to the ferret superior colliculus: anatomical contribution to the neural coding of sound azimuth. J Comp Neurol 390:342-365.

Linke R, Schwegler H (2000) Convergent and complementary projections of the caudal paralaminar thalamic nuclei to rat temporal and insular cortex. Cereb Cortex 10:753-771.

Malmierca MS, Oliver D, Henkel C, Merchán MA (2002) A novel projection from the dorsal cochlear nucleus to the medial division of the medial geniculate body in rat. ARO Abstr 22:667.

Merchán MA, Saldaña E, Plaza I (1994) Dorsal nucleus of the lateral lemniscus in the rat: concentric organization and tonotopic projection to the inferior colliculus. J Comp Neurol 342:259-278.

Morest DK (1965) The lateral tegmental system of the midbrain and the medial geniculate body: study with Golgi and Nauta methods in cat. J Anat 99:611-634.

Morest DK, Oliver DL (1984) The neuronal architecture of the inferior colliculus in the cat: defining the functional anatomy of the auditory midbrain. J Comp Neurol 222:209-236.

Ohlrogge M, Doucet JR, Ryugo DK (2001) Projections of the pontine nuclei to the cochlear nucleus in rats. J Comp Neurol 436:290-303.

Oliver DL (1985) Quantitative analyses of axonal endings in the central nucleus of the inferior colliculus and distribution of $3 \mathrm{H}$-labeling after injections in the dorsal cochlear nucleus. J Comp Neurol 237:343-359.

Oliver DL, Hall WC (1978) The medial geniculate body of the tree shrew, Tupaia glis. II. Connections with the neocortex. J Comp Neurol 182:459-493.

Oliver DL, Beckius GE, Bishop DC, Kuwada S (1997) Simultaneous anterograde labeling of axonal layers from lateral superior olive and dorsal cochlear nucleus in the inferior colliculus of cat. J Comp Neurol 382:215-229.

Osen KK (1969) Cytoarchitecture of the cochlear nuclei in the cat. J Comp Neurol 136:453-484.

Palombi PS, Caspary DM (1996) Physiology of the young adult Fischer 344 rat inferior colliculus: responses to contralateral monaural stimuli. Hear Res 100:41-58.

Ramón y Cajal S (1902) Estructura del tubérculo cuadrigémino posterior, cuerpo geniculado interno y vías acústicas centrales. Rev Tri Micrográf 6:207-227.
Rees A, Moller AR (1983) Responses of neurons in the inferior colliculus of the rat to AM and FM tones. Hear Res 10:301-330.

Rees A, Sarbaz A, Malmierca MS, Le Beau FE (1997) Regularity of firing of neurons in the inferior colliculus. J Neurophysiol 77:2945-2965.

Rouiller EM, Welker E (1991) Morphology of corticothalamic terminals arising from the auditory cortex of the rat: a Phaseolus vulgarisleucoagglutinin (PHA-L) tracing study. Hear Res 56:179-190.

Rouiller EM, Wan XST, Moret V, Liang F (1992) Mapping of c-fos expression elicited by pure tones stimulation in the auditory pathways of the rat, with emphasis on the cochlear nucleus. Neurosci Lett 144:19-24.

Ryan AF, Furlow Z, Woolf NK, Keithley EM (1988) The spatial representation of frequency in the rat dorsal cochlear nucleus and inferior colliculus. Hear Res 36:181-189.

Ryugo DK, Killackey HP (1974) Differential telencephalic projections of the medial and ventral divisions of the medial geniculate body of the rat. Brain Res 82:173-177.

Shiroyama T, Kayahara T, Yasui Y, Nomura J, Nakano K (1999) Projections of the vestibular nuclei to the thalamus in the rat: a Phaseolus vulgaris leucoagglutinin study. J Comp Neurol 407:318-332.

Shore SE, Vass Z, Wys NL, Altschuller RA (2000) Trigeminal ganglion innervates the auditory brainstem. J Comp Neurol 419:271-285.

Smith PH, Spirou G (2002) From the cochlea to the cortex and back. In: Mammalian auditory pathways: structure and function (Oertel D, Fay RR, Popper AN, eds), pp 6-71. New York: Springer-Verlag.

Strominger NL, Nelson LR, Dougherty WJ (1977) Second order auditory pathways in the chimpanzee. J Comp Neurol 172:349-365.

Weedman DL, Ryugo DK (1996) Pyramidal cells in primary auditory cortex project to cochlear nucleus in rat. Brain Res 706:97-102.

Weinberg RJ, Rustioni A (1987) A cuneocochlear pathway in the rat. Neuroscience 20:209-219.

Wepsic JG (1966) Multimodal sensory activation of cells in the magnocellular medial geniculate nucleus. Exp Neurol 15:299-318.

Winer JA, Larue DT (1987) Patterns of reciprocity in auditory thalamocortical and corticothalamic connections: study with horseradish peroxidase and autoradiographic methods in the rat medial geniculate body. J Comp Neurol 257:282-315.

Wright DD, Ryugo DK (1996) Mossy fiber projections from the cuneate nucleus to the cochlear nucleus in the rat. J Comp Neurol 365:159-172.

Young ED, Nelken I, Conley RA (1995) Somatosensory effects on neurons in dorsal cochlear nucleus. J Neurophysiol 73:743-765.

Yu JJ, Young ED (2000) Linear and nonlinear pathways of spectral information transmission in the cochlear nucleus. Proc Natl Acad Sci USA 97:11780-11786. 